\title{
THE DITTERT'S FUNCTION ON A SET OF NONNEGATIVE MATRICES
}

\author{
SUK GEUN HWANG and MUN-GU SOHN \\ Department of Mathematics \\ Teachers College \\ Kyungpook University \\ Taegu 702-701 \\ Republic of Korea \\ SI-JU KIM \\ Department of Mathematics Education \\ Andong University \\ Andong, Kyungpook 760-380 \\ Republic of Korea \\ (Received November 6, 1989 and in revised form January 12, 1990)
}

ABSTRACT. Let $K_{n}$ denote the set of all $n \times n$ nonnegative matrices with entry sum $n$. For $X \in K_{n}$ with row sum vector $\left(r_{1}, \ldots, r_{n}\right)$, column sum vector $\left(c_{1}, \ldots, c_{n}\right)$, Let $\phi(X)=\pi r_{i}+\pi c_{j}-$ perX. Dittert's conjecture asserts that $\phi(X)<2-n ! / n^{n^{n}}$ for all $X \frac{1}{\varepsilon} K_{n}^{1}$ with equality iff $X=[1 / n]_{n \times n}$. This paper investigates some properties of a certain subclass of $k_{n}$ related to the function $\phi$ and the Dittert's conjecture.

KEY WORDS AND PHRASES. Permanent, Dittert's function, $\Lambda$-admissable matrix.

1980 AMS SUBJECT CLASSIFICATION CODE. 15 A48.

1. INTRODUCTION.

Let $K_{n}$ denote the set of all $n \times n$ nonnegative matrices whose entries have sum $n$, and let $\phi$ denote a real valued function of $k_{n}$ defined by

$$
\phi(x)=\prod_{i=1}^{n} \sum_{j=1}^{n} x_{i j}+\prod_{j=1}^{n} \sum_{i=1}^{n} x_{i j}-\operatorname{per} x
$$

for $X=\left[x_{i j}\right] \varepsilon K_{n}$ where perX stands for the permanent of $X$;

$$
\operatorname{perX}=\sum_{\sigma \varepsilon S_{n}} x_{1 \sigma(1)} \cdots x_{n \sigma(n)}
$$

Let $J_{n}$ denote the $n \times n$ matrix all of whose entries are $1 / n$. For the function $\phi$ there is a conjecture due to Eric Dittert.

CONJECTURE (Marcus and Merris [1], Conjecture 28]). For $A \varepsilon K_{n}$,

$$
\phi(A)<2-\frac{n !}{n^{n}}
$$

with equality if and only if $A=J_{n}$. 
In this paper, we will call $\phi$ the Dillerl's function. It is proved that the Dittert's conjecture is true for $n \leqslant 3$ (Marcus and Merris [1], Sinkhorn [2], and Hwang [3]). For a matrix $X \in K_{n}$ whose row sum vector is $\left(r_{1}, \ldots, r_{n}\right)$ and whose column sum vector is $\left(c_{1}, \ldots, c_{n}\right)$,

Let

$$
\begin{aligned}
& \bar{r}_{i}=r_{1} \ldots r_{i-1} r_{i+1} \cdots r_{n}(i=1, \ldots, n), \\
& \bar{c}_{j}=c_{1} \cdots c_{j-1} c_{j+1} \cdots c_{n}(j=1, \ldots, n)
\end{aligned}
$$

and

$$
\phi_{i j}(x)=\bar{r}_{j}+\bar{c}_{j}-\operatorname{perx}(i \mid j) \quad(i, j=1,2, \ldots, n)
$$

where $x(1 \mid j)$ denotes the matrix obtained from $x$ by deleting the row 1 and column $j$. A matrix $A \varepsilon K_{n}$ is called a $\phi$ - maximizing matrix on $K_{n}$ if $\phi(A)>\phi(X)$ for all $X \in K_{n}$. In [3], the following results are proved.

THEOREM A. If $A=\left[a_{i j}\right]$ is a $\phi$ - maximizing matrix on $K_{n}$, then

$$
\phi_{1 j}(A) \begin{cases}=\phi(A) & \text { if } a_{i j}>0 \\ <\phi(A) & \text { if } a_{1 j}=0 .\end{cases}
$$

THEOREM B. If, for every $\phi$ - maximizing matrix $A$ on $K_{n}, \phi_{1 j}(A)=\phi(A)$ for all $1, j=1, \ldots, n$, then $J_{n}$ is the unique $\phi-$ maximizing matrix on $K_{n}$.

We see that $\phi(A)>0$ for all $A \in K_{n}$. For $A \in K_{n}$ with row sum vector $\left(r_{1}, \ldots, r_{n}\right)$ and column sum vector $\left(c_{1}, \ldots, c_{n}\right)$, if either $r_{1} \ldots r_{n}>0$ or $c_{1} \ldots c_{n}>0$, then $\phi(A)>0$. Now, for $A \varepsilon k_{n}$ with $\phi(A)>0$, Let $A^{*}=\left[a_{1 j}^{*}\right]$ denote the $n \times n$ matrix defined by

$$
a_{1 j}^{\star}=\frac{\phi_{1 j}(A)}{\phi(A)} \quad(1, j=1, \ldots, n) .
$$

For $\Lambda \varepsilon K_{n}$, we say that $A \varepsilon K_{n}$ with $\phi(A)>0$ is $\Lambda$-admissable (or $A$ is admissable by $\Lambda$ ) if $\operatorname{tr}\left(\Lambda_{A}^{T}\right)>n$ where $\Lambda^{T}$ denotes the transpose of $\Lambda$ and $t r$ denotes the trace function. Let $\zeta_{i}(\Lambda)$ denotes the set of all $\Lambda$-admissable matrices.

It follows from Theorem $A$ that every $\phi$-maximizing matrix $A$ is self-admissable i.e. $A \in \zeta(A)$.

If for each $\phi$-maximizing matrix $A$ there exists a positive matrix $\Lambda \varepsilon K_{n}$ such that $A \in P(\Lambda)$, then the Dittert's conjecture is true (See section 2).

In such a point of view, it would be interesting to study the classes ( $\Lambda$ ) for some particular matrices $\Lambda \varepsilon K_{n}$. Such a matrix $\Lambda$ should be one which is most likely to posess the property that all $\phi$-maximizing matrices on $K_{n}$ are $\Lambda$-admissable.

In this paper we find some matrices in $C_{\infty}(\Lambda)$ for certain $\Lambda^{\prime} s$ and investigate some properties of the Dittert's function related to the class $:(\Lambda)$.

2. THE Class $f_{i}(\Lambda)$ and $\phi$ - Maximizing matrices.

From now on let $\operatorname{Max}\left(K_{n}\right)$ denote the set of all $\phi$-maximizing matrices on $K_{n}$. 
THEOREM 2.1. If each $A \varepsilon \operatorname{Max}\left(K_{n}\right)$ is admissable by a positive matrix in $K_{n}$, then $\operatorname{Max}\left(K_{n}\right)=\left\{J_{n}\right\}$, i.e. the Dittert's conjecture holds.

PROOF. Let $A \in \operatorname{Max}\left(K_{n}\right)$ and let $\Lambda=\left[\lambda_{i j}\right] \& K_{n}$ be a positive matrix such that $A \in C(\Lambda)$. Then

$$
n<\operatorname{tr}\left(\Lambda^{T} A^{*}\right)=\sum_{i=1}^{n} \sum_{j=1}^{n} \lambda_{i j} \frac{\phi_{i j}(A)}{\phi(A)}<\sum_{i=1}^{n} \sum_{j=1}^{n} \lambda_{i j}=n
$$

by Theorem $A$. Therefore the inequalitites in (2.1) are all equalities and hence

$\phi_{1 j}(A)=\phi(A)$ for all $i, j=1,2, \ldots, n$ since $\Lambda$ is a positive matrix. Now the assertion of the theorem follows from Theorem $B$.

For $A \varepsilon K_{n}$ with row sum vector $\left(r_{1}, \ldots, r_{n}\right)$ and column sum vector $\left(c_{1}, \ldots, c_{n}\right)$, Let $A=\left[a_{i j}\right]$ denote the $n \times n$ matrix defined by

$$
\hat{a}_{i j}=\frac{r_{i} c_{j}}{n}(i, j=1, \ldots, n) \text {. }
$$

Since $\sum_{i=1}^{n} \sum_{i=1}^{n} r_{i} c_{j}=n^{2}$ we see that $\dot{A} \varepsilon K_{n}$. In particular if $A \in \operatorname{Max}\left(K_{n}\right)$, then $\ddot{A}$ is a positive matrix since $r_{i}>0, c_{j}>0$ for all $1, j=1, \ldots, n$ because perA $>0$ [2].

We believe that every $A \in \operatorname{Max}\left(K_{n}\right)$ is $\grave{A}$-admissable, which we can not prove yet. We may ask which matrices $A \in K_{n}$ are $\hat{A}^{-}$-admisable and which are not. We have an answer to this question.

THEOREM 2.2. If A is positive semidefinite symmetric matrix in $K_{n}$, then $A \underline{A}$ admissable.

PROOF. Let $A$ be a p.s.d. symmetric matrix in $K_{n}$ and let $r_{1}$ be the 1 -th row sum of $A(1=1, \ldots, n)$. Then the condition that $A$ is $\hat{A}$-admissable is equivalent to

$$
\sum_{i=1}^{n} \sum_{j=1}^{n} r_{i} r_{j} \phi_{i j}(A)>n^{2} \phi(A) \text {. }
$$

Let $r=r_{1} \ldots r_{n}$ and let $\bar{r}_{1}=r_{1} \ldots r_{1-1} r_{1+1} \ldots r_{n}(1=1, \ldots, n)$. Then

$$
\begin{aligned}
\sum_{i=1}^{n} \sum_{j=1}^{n} r_{i} r_{j} \phi_{1 j}(A) & =\sum_{i=1}^{n} \sum_{j=1}^{n} r_{i} r_{j}\left(\bar{r}_{i}+\bar{r}_{j}-\operatorname{perA}(I \mid j)\right] \\
& =\sum_{i=1}^{n} \sum_{j=1}^{n}\left[\left(r_{i}+r_{j}\right) r-r_{i} r_{j} \operatorname{perA}(i \mid j)\right] \\
& =2 n^{2} r-\sum_{i=1}^{n} \sum_{j=1}^{n} r_{i} r_{j} \operatorname{perA}(i \mid j) .
\end{aligned}
$$

Since

$$
\sum_{1=1}^{n} \sum_{j=1}^{n} r_{1} r_{j} \operatorname{perA}(1 \mid j)<n^{2} \operatorname{perA}
$$

by a theorem of Marcus and Merris [4], we have 


$$
\sum_{i=1}^{n} \sum_{i=1}^{n} r_{i} r_{j} \phi_{i j}(A)>2 n^{2} r-n^{2} \operatorname{per} A=n^{2} \phi(A)
$$

and the proof is complete.

Note that not every matrix $A \in K_{n}$ is $\widehat{A}$-admissable. For $n=2$, the matrix

$$
A_{x}=\left[\begin{array}{ll}
2-2 x & x \\
x & 0
\end{array}\right]
$$

in $K_{2}$ is not $\hat{A}_{x}$-admissable if $0<x<1 / 2$. For $n>3$, we have an EXAMPLE 2.1. Let $T_{n}$ denote the following $n \times n$ matrix.

$$
T_{n}=\left[\begin{array}{cccc}
0 & \frac{1}{n-1} & \cdots & \frac{1}{n-1} \\
\vdots & \vdots & & \vdots \\
0 & \frac{1}{n-1} & \cdots & \frac{1}{n-1} \\
1 & 0 & \cdots & 0 \\
1 & 0 & \cdots & 0
\end{array}\right] .
$$

Then $T_{n} \varepsilon K_{n}$ and $\left(r_{1}, \ldots, r_{n}\right)=(1, \ldots, 1),\left(c_{1}, \ldots, c_{n}\right)=\left(2, \frac{n-2}{n-1}, \ldots, \frac{n-2}{n-1}\right) . \quad$ We have

$$
n^{2} \phi\left(T_{n}\right)-\sum_{i=1}^{n} \sum_{j=1}^{n} r_{1} c_{j} \phi_{i j}\left(T_{n}\right)=2 \frac{(n-1) !}{(n-1)^{n-2}}>0
$$

so that $T_{n} \in \zeta\left(T_{n}\right)$ and hence that $T_{n}$ is not $\hat{T}_{n}$-admissable.

3. THE CLASS $\ell\left(J_{n}\right)$ AND THE MONOTONICITY OF THE DitTERT'S FUnCtion.

Another candidate for positive $\Lambda \varepsilon K_{n}$ with "good" $\zeta_{i}(\Lambda)$ is the matrix $J_{n}$. A nonnegative square matrix is called a doubly stochastic matrix if all the row sums and column sums are equal to 1 . It is conjectured that every $n \times n$ doubly stochastic matrix is $J_{n}$-admissable (Dokovic [5] and Minc [6]) but this still remains open. Here we have to notice that $A$ is $J_{n}$-admissable (1.e. A $\varepsilon \ell_{f}\left(J_{n}\right)$ ) if and only if

$$
\sum_{i=1 j=1}^{n} \sum_{i j}^{n}(A)>n^{2} \phi(A) \text {. }
$$

We can show that $\dot{\zeta}_{\left(J_{n}\right) \neq K_{n}}$ for $n>3$ (see Example 3.1). However it seems that $\operatorname{Max}\left(K_{n}\right) \quad\left(J_{n}\right)$. It is clear that $J_{n}$ and the $n \times n$ identity matrix $I_{n}$ are $J_{n}-$ admissable. We can show that all diagonal matrices in $K_{n}$ are also $J_{n}$-admissable.

THEOREM 3.1. Every diagonal matrix in $K_{n}$ is $J_{n}$-admissable.

PROOF. Let $A=\operatorname{diag}\left(a_{1}, \ldots, a_{n}\right) \varepsilon K_{n}, a=a_{1} \ldots a_{n}$ and $\bar{a}_{1}=a_{1} \ldots a_{1-1}$ $a_{1+1} \cdots a_{n}(1=1, \ldots, n)$. If $a=0$, there is nothing to prove. Suppose a $>0$. Then $\phi(A)=a$ and

$$
\sum_{i=1}^{n} \sum_{i=1}^{n} \phi_{i j}(A)=\sum_{i=1}^{n} \sum_{j=1}^{n}\left(\bar{a}_{i}+\bar{a}_{j}\right)-\sum_{i=1}^{n} \bar{a}_{i}
$$




$$
\begin{aligned}
& =(2 n-1) \sum_{i=1}^{n} \bar{a}_{i} \\
& =(2 n-1) a \sum_{i=1}^{n} \frac{1}{a_{i}}>n(2 n-1) a .
\end{aligned}
$$

Therefore,

$$
\phi(A)<\frac{1}{n^{2}} \sum_{i=1}^{n} \sum_{j=1}^{n} \phi_{i j}(A)
$$

if $\mathrm{n}>2$, and the proof is complete.

The Dittert's function $\phi$ has some nice behavior on the set $\phi_{p}\left(J_{n}\right)$ namely that $\phi$ is monotone on the straight line segment joining $J_{n}$ and $A \varepsilon i_{p}^{k_{j}}\left(J_{n}\right)$ whenever the line segment lies in $\zeta_{(}\left(J_{n}\right)$. To show this, let $\Delta$ be a function define by

$$
\Delta(X)=\phi(X)-\frac{1}{n^{2}} \sum_{i=1 j=1}^{n} \sum_{1 j}^{n} \phi_{1}(x), x \in K_{n} .
$$

Let $A=\left[a_{i j}\right] \in K_{n}$ have row sum vector $\left(r_{1}, \ldots, r_{n}\right)$ and column sum vector $\left(c_{1}, \ldots, c_{n}\right)$. For a real number $t, 0<t<1$, let $A_{t}=(1-t) J_{n}+t A:=\left[a_{1 j}(t)\right]$ and let the row sum vector and the column sum vector of $A_{t}$ be $\left(r_{1}(t), \ldots, r_{n}(t)\right)$ and $\left(c_{1}(t), \ldots, c_{n}(t)\right)$ respectively.

Letting

$$
\begin{aligned}
& r(t)=r_{1}(t) \ldots r_{n}(t), \\
& c(t)=c_{1}(t) \ldots c_{n}(t), \\
& \bar{r}_{1}(t)=r_{1}(t) \ldots r_{i-1}(t) r_{i+1}(t) \ldots r_{n}(t), \quad(i=1, \ldots, n), \\
& \bar{c}_{j}(t)=c_{1}(t) \ldots c_{j-1}(t) c_{j+1}(t) \ldots c_{n}(t),(j=1, \ldots, n),
\end{aligned}
$$

we compute, for $t>0$, that

so that

$$
\begin{aligned}
\frac{d}{d t} r(t) & =\frac{1}{t} \sum_{i=1}^{n}\left\{r(t)-\bar{r}_{i}(t)\right\} \\
& =\frac{n}{t}\left\{r(t)-\frac{1}{n^{2}} \sum_{i=1}^{n} \sum_{j=1}^{n} \bar{r}_{i}(t)\right\}, \\
\frac{d}{d t} c(t) & =\frac{1}{t} \sum_{j=1}^{n}\left\{c(t)-\bar{c}_{j}(t)\right\} \\
& =\frac{n}{t}\left\{c(t)-\frac{1}{n^{2}} \sum_{j=1}^{n} \bar{c}_{j}(t)\right\}, \\
\frac{d}{d t} \operatorname{perA}_{t} & =\frac{n}{t}\left\{\operatorname{perA} A_{t}-\frac{1}{n^{2}} \sum_{i=1}^{n} \sum_{j=1}^{n} \operatorname{perA}_{t}(i \mid j)\right\}
\end{aligned}
$$

$$
\begin{aligned}
\frac{d}{d t} \phi\left(A_{t}\right) & =\frac{n}{t}\left\{r(t)+c(t)-\operatorname{perA}_{t}\right. \\
& \left.-\frac{1}{n^{2}} \sum_{i=1}^{n} \sum_{j=1}^{n}\left[\bar{r}_{i}(t)+\bar{c}_{j}(t)-\operatorname{perA}_{t}(i \mid j)\right]\right\}
\end{aligned}
$$




$$
=\frac{n}{t}\left\{\phi\left(A_{t}\right)-\frac{1}{n^{2}} \sum_{i=1 j=1}^{n} \sum_{i j}^{n} \phi_{i j}\left(A_{t}\right)\right\},
$$

which is

Thus we have the following

$$
\frac{d}{d t} \phi\left(A_{t}\right)=\frac{n}{t} \Delta\left(A_{t}\right) .
$$

THEOREM 3.2. Let $A \in \mathrm{K}_{\mathrm{n}}$. If $\mathrm{A}_{\mathrm{t}} \varepsilon \mathrm{C}\left(\mathrm{J}_{\mathrm{n}}\right)$ for all $\mathrm{t}, 0<\mathrm{t}<1$, then the Dirrert's function is monotone decreasing on the straight line segment from $J_{n}$ to $A$.

It is not hard to show that, for any $A \in K_{2}$,

$$
\frac{1}{2^{2}} \sum_{i=1}^{2} \sum_{j=1}^{2} \phi_{i j}(A)=\frac{3}{2} \text {. }
$$

On the other hand, the validity of Dittert's conjecture for $n=2$ gives us that

$$
\frac{3}{2}=\phi\left(J_{n}\right)>\phi(A) .
$$

Therefore it follows that $\mathrm{K}_{2}=\mathrm{C}\left(\mathrm{J}_{2}\right)$. However it does not hold in general thạt $\mathrm{K}_{\mathrm{n}}=\mathrm{C}\left(\mathrm{J}_{\mathrm{n}}\right)$.

EXAMPLE 3.1. Let

$$
U_{n}=\left[\begin{array}{cccc}
\frac{n}{n+1} & \frac{n}{n+1} \\
& \frac{n}{n+1} & 0 & 0 \\
\frac{n}{n+1} & 0 & & \\
\frac{n}{n+1} & 0 & 0
\end{array}\right], n \geqslant 4
$$

and let

$$
u_{3}=\left[\begin{array}{ccc}
0 & \frac{3}{4} & \frac{3}{4} \\
\frac{3}{4} & 0 & 0 \\
\frac{3}{4} & 0 & 0
\end{array}\right] .
$$

Then

$$
\phi\left(u_{n}\right)=4\left(\frac{n}{n+1}\right)^{n}
$$

and 


$$
U_{n}^{*}=\frac{n+1}{4 n}\left[\begin{array}{cccccc}
2 & 3 & \cdots & 3 & 3 \\
3 & 4 & . & 4 & 3 & 3 \\
\vdots & \vdots & \vdots & \vdots & \vdots \\
3 & 3 & \cdots & 3 & 3 & 3 \\
3 & 3 & 3 & 3 & 3
\end{array}\right]
$$

Hence

$$
\begin{aligned}
& \sum_{i=1}^{n} \sum_{j=1}^{n} \phi_{1 j}\left(U_{n}\right) \\
& =\phi\left(U_{n}\right) \times\left(\text { sum of entries of } U_{n}^{*}\right) \\
& =4\left(\frac{n}{n+1}\right)^{n} \frac{n+1}{4 n}\left[2+4(n-3)^{2}+3(6 n-10)\right] \\
& =\left(\frac{n}{n+1}\right)^{n-1}\left(4 n^{2}-6 n+8\right) .
\end{aligned}
$$

Thus we have

$$
\begin{aligned}
& n^{2} \phi\left(U_{n}\right)-\sum_{i=1}^{n} \sum_{j=1}^{n} \phi_{i j}\left(U_{n}\right) \\
& =\left(\frac{n}{n+1}\right)^{n-1}\left(\frac{4 n^{3}}{n+1}-4 n^{2}+6 n-8\right) \\
& =\frac{n^{n-1}}{(n+1)^{n}}\left(2 n^{2}-2 n-8\right)
\end{aligned}
$$

which is positive for all $n>3$, telling us that $U_{n}$ is not $J_{n}$-admissable.

4. CONCLUDING REMARKS.

If, for every $A \varepsilon \operatorname{Max}\left(K_{n}\right)$, we could find a positive matrix $\Lambda \in K_{n}$ such that $A$ is admissable by $\Lambda$, it would prove the Dittert's confecture by Theorem 2.1 . It seems to us that the matrices $\hat{A}$ or $J_{n}$ are two of the strongest candidates for such matrices. However we may not expect to have a positive matrix $\Lambda \in K_{n}$ such that all the matrices in $K_{n}$ are $\Lambda$-admissable.

We shall close our discussion here by giving some further research problems.

PROBLEM 4.1. Determine whether there exists a positive matrix $\Lambda \varepsilon K_{n}$ admitting all matrices in $K_{n}$.

We conjecture that such a matrix does not exist.

It is proved that every p.s.d. symmetric doubly stochastic matrix is $J_{n}-$ admissable [4], from which it follows that the permanent function is monotone increasing on the straight line sequment from $J_{n}$ to any p.s.d. symmetric doubly stochastic matrix (Hwang [7]).

PROBLEM 4.1. Determine whether every p.s.d. symmetric matrix in $K_{n}$ is $J_{n}-$ admissable. 
If every p.s.d. symmetric matrix in $K_{n}$ is $J_{n}$-admissable, then it follows from Theorem 3.2 that the Dittert's function is monotone decreasing on the straight line segment from $J_{n}$ to any p.s.d. symmetric matrix in $K_{n}$. We conjecture that the Problem 4.1 will have an affirmative answer.

PROBLEM 4.3. Is every $\phi$-maximizing matrix $A$ on $K_{n} \hat{A}$-admissable or $J_{n}$-admissable?

If Problem 4.3 has an a affirmative answer, it would prove the Dittert's conjecture as we stated earlier.

ACKNOWLEDGEMENT. Research supported by a grant from the Korean Ministry of Eduation via the Kyungpook University Basic Science Res. Inst., 1988.

\section{REFERENCES}

1. MINC, H. Theory of permanents 1982-1985, Lin. Multilin. Alg. 12 (1982).

2. SINKHORN, R. A problem related to the van der Waerden permanent theorem, Lin. Multilin. Alg. 16(1984), 167-173.

3. HWANG, S.G. On a conjecture of E. Dittert, Lin. Alg. App1. 95 (1987), 161-169.

4. MARCUS, M. and MERRIS, R. A relation between permanental and determinantal adjoints, J. Austral. Math. Soc. 15 (1973), 270-271.

5. DOKOVIC, D.Z. On a conjecture by van der Waerden, Mat. Vesnik 19(4) (1967, 272276.

6. MINC, H. Theory of permanents 1978-1981, Lin. Mult1lin. Alg. 12 (1982), 227-263.

7. HWANG, S.G. On the monotonicity of the permanent, to appear in Proc. Amer.

8. MINC, H. Permanents, Encyclopedia of Math. and Its Appl. 6, Addison-Wesley, 1978.

9. HWANG, S.G. A note on a conjecture on permanents, Lin. Alg. Appl. 76 (1986), 3144.

The present address of Dr. Suk Geun Hwang is:

Department of Mathematics

Sung Kyun Kwan University

Suwon 440-746

Republic of Korea 


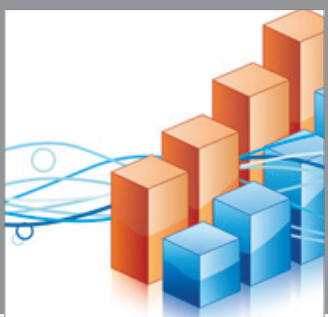

Advances in

Operations Research

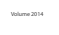

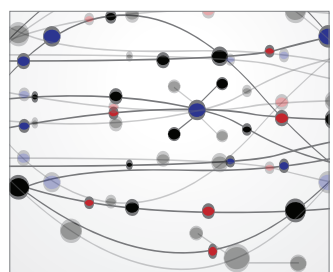

\section{The Scientific} World Journal
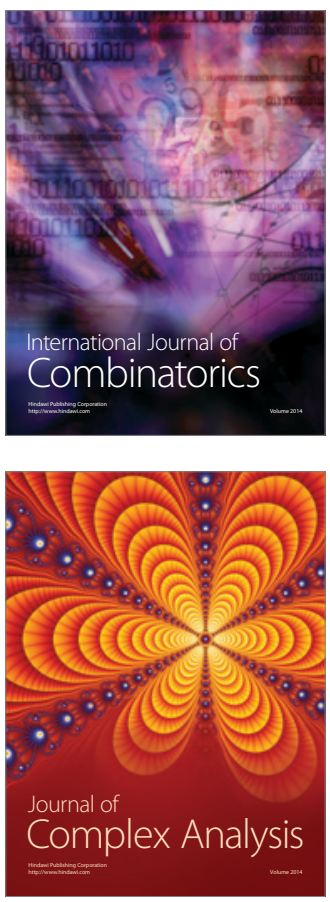

International Journal of

Mathematics and

Mathematical

Sciences
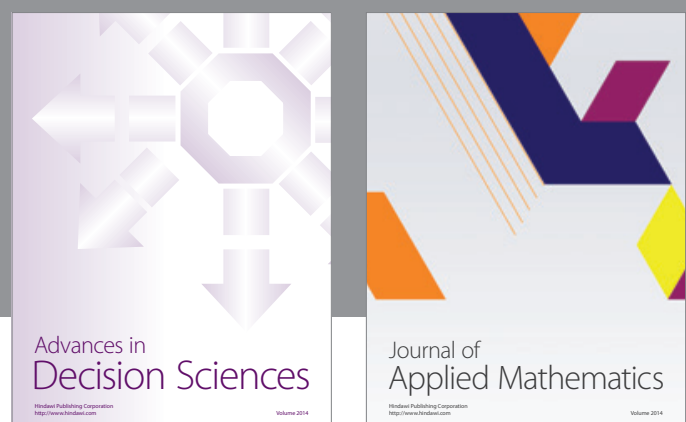

Journal of

Applied Mathematics
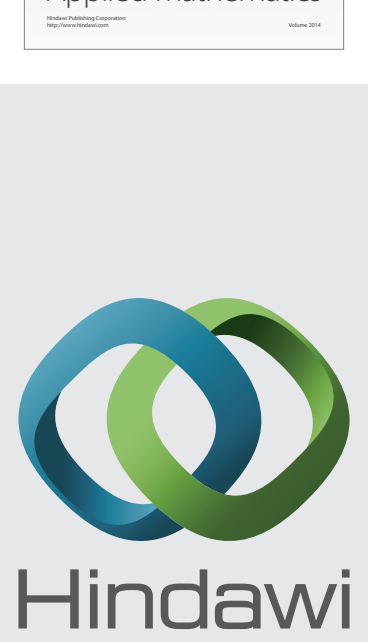

Submit your manuscripts at http://www.hindawi.com
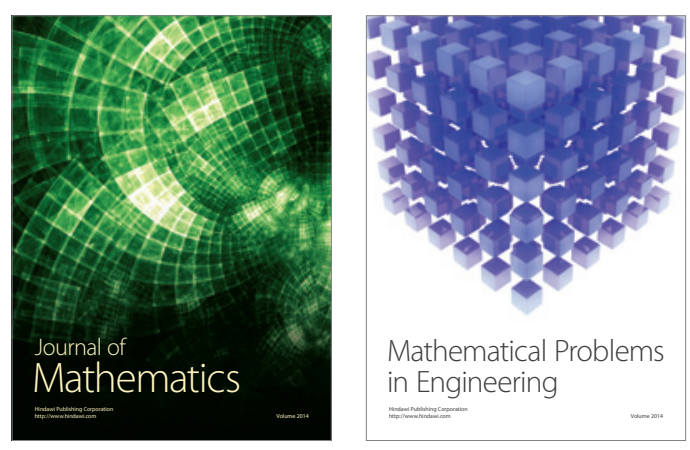

Mathematical Problems in Engineering
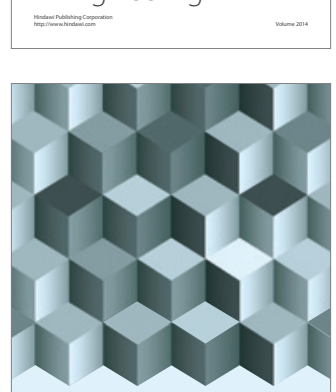

Journal of

Function Spaces
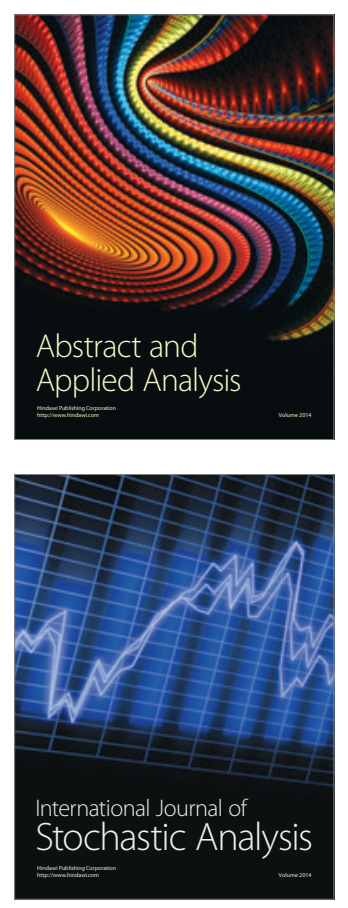

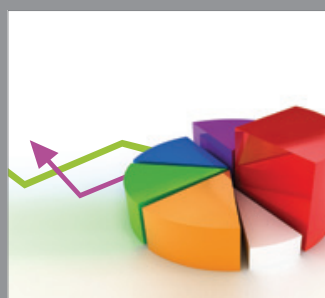

ournal of

Probability and Statistics

Promensencen
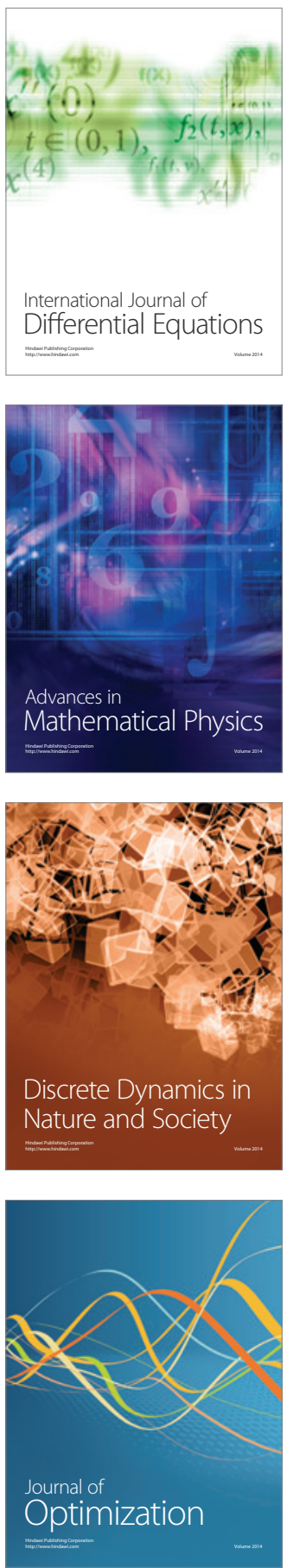